\title{
The neglected tropical diseases (NTD) initiative for Latin America and the Caribbean of the Inter-American Development Bank and the role of geospatial analysis in health programmes
}

\author{
Josh Colston \\ Social Protection and Health Division, Inter-American Development Bank, Washington DC, USA
}

What is the Inter-American Development Bank?

The Inter-American Development Bank (IDB) (http://www.iadb.org/en/inter-american-developmentbank,2837.html) is the regional, multilateral development bank for Latin America and the Caribbean (LAC). The largest source of external development financing for the region, the IDB support efforts by LAC countries to reduce poverty and inequality with the aim of bringing about development in a sustainable, climate-friendly way. Of the IDB's 48 member states, 26 are borrowing countries which own majority shares in the institution. In 2010, the IDB approved an unprecedented 170 projects and US\$ 570 million in grant financing. The IDB's Social Protection and Health (SPH) division falls under the social sector and has a total of 56 staff working in the fields of health and social protection with the majority based in country offices of the borrowing countries themselves. The SPH division's priorities in social protection are:

(i) early childhood development (ECD), with focus on infants up to 3 years of age;

(ii) youth at risk (in coordination with other business units); and

(iii) poverty alleviation programmes.

Its priorities in health are:

(i) accountability, efficiency and effectiveness of health systems; and

(ii) strengthening health networks with emphasis on primary care for underserved communities.

Corresponding author:

Josh Colston

Technical Adviser to the LAC NTD Initiative, Social Protection

and Health Division, Inter-American Development Bank

1300 New York Ave. NW Washington, DC 20577, USA

Tel. +12026232496

E- mail: joshcolston@gmail.com
The neglected tropical diseases (NTDs) in LAC

The NTD situation in LAC is unique. While in other regions they are often widespread across large areas, the distribution in LAC of many NTDs is concentrated in small niches - stubborn pockets of transmission where the conditions are just right for the diseases to survive, continue infecting people and resist attempts to tackle them. Cheap and safe medicines can quickly rid people of NTD infections. These are regularly given out on a massive scale in NTD-endemic countries around the world, despite the fact that these medicines are shortterm curative measures. Unless the conditions that perpetuate the transmission are tackled, the diseases soon come back. A cured individual that goes back to the same environment where they originally caught the disease will soon become re-infected. A long-term, sustainable solution to the NTD problem therefore has to focus on improving the living conditions that put people at risk. Unfortunately, holistic approaches like this are costly and require a lot of coordination between different sectors - health, education, housing and, importantly, water and sanitation. Fortunately, however, another way in which LAC is unique is that the elimination of several NTDs - trachoma, onchocerciasis and lymphatic filariasis (LF) among them - is a genuinely feasible goal in the short term.

\section{IDB's work with respect to the NTDs}

Recognizing the achievability of control and eventually elimination of NTDs in the Americas, the IDB has entered into a partnership with the Global Network for NTDs, (Global Network) (http://www.globalnetwork.org/) and the Pan American Health Organization (PAHO) (http://new.paho.org/index .php) to establish an initiative that is providing technical and financial support for the control and elimination of NTDs in LAC. The aim of this partnership is to support projects that employ an integrated, com- 
munity-based methodology that goes beyond shortterm curative measures to include longer-term solutions that tackle the social and environmental determinants of disease transmission. The partnership aims to coordinate and support country ownership and capacity building to address the burden of NTDs in the region. Targeted at national governments, national NTD programmes, and non-governmental organizations, the partnership promotes the integration of NTD prevention, control and elimination activities into existing systems and efforts, such as primary care, clean water and improved sanitation initiatives, conditional cash transfer programmes and housing initiatives. It acts as a regional hub to facilitate the development of an NTD agenda for LAC, while developing priorities for integrated disease control, project implementation, advocacy and resource mobilization. As the regional development bank for LAC, with projects in many different sectors, one of the things that the IDB brings to the partnership is the ability to facilitate this integration. We can collaborate with our colleagues in different departments, whose projects tackle NTD risk factors to include effective, low-cost public health activities within their projects. All NTD projects carried out by the IDB will be guided by the following five principles:

(i) national planning to support inter- and intra-sectoral coordination for NTD control;

(ii) mapping and technical assistance to strengthen national and local health surveillance for NTDs;

(iii) community mobilization and education;

(iv) preventative and curative treatment for NTDs; and

(v) systematization, monitoring and evaluation.

\section{Ongoing projects}

\section{Mexico, Chiapas}

Chiapas is one of the poorest states of Mexico with a relatively dispersed and marginalized population many of whom live in remote settlements with poor amenities. These conditions make the state one of the major hotspots for NTD transmission in the LAC region. The focus of the IDB's project here is on building the capacity within the health system and personnel to provide complete and timely referral and treatment of the diseases along with integrated surveillance and community mobilization. The high levels of government commitment for improving this situation particularly in the areas of water and sanitation and the elimination of trachoma will ensure success. The 132,000 inhabitants of the five selected municipalities

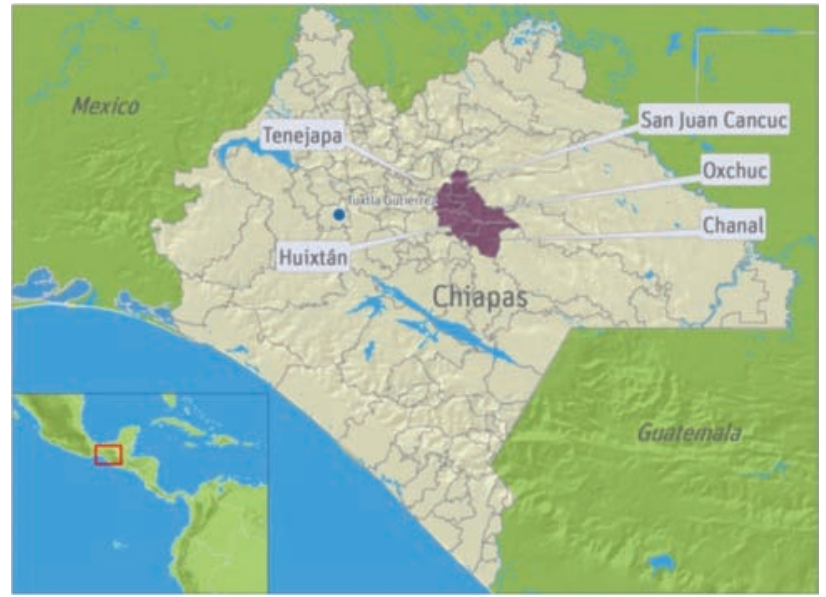

Fig. 1. IDB project RG-X1051: neglected diseases in Chiapas, México: a demonstration project.

(Fig. 1) will benefit directly, while the entire population of Chiapas - more than 4 million people - will benefit indirectly from the activities.

\section{Brazil, Recife Metropolitan area}

Recife is one of the largest cities in Brazil and one that suffers from considerable inequality with pockets of extreme deprivation. The city is notable for being the one location in Brazil where transmission of LF continues. The project here aims to build on the political will towards national LF elimination to integrate activities for the control of NTDs, such as mass drug administration (MDA) for soil-transmitted helminths (STHs) and LF and selective treatment of schistosomiasis and leprosy. These interventions will be strengthened through the involvement of the community and integration with water and sanitation strategies. In this project, the 154,000 inhabitants will benefit directly, while the entire population of the three municipalities in the Recife metropolitan area (Fig. 2) targeted for intervention - around 2.6 million people - will benefit indirectly from the activities.

\section{Guatemala, two departments}

Approximately $51 \%$ of Guatemalans live below the poverty line, and $15 \%$ in extreme poverty. Almost 3 million still have no water services and approximately 6 million are without sanitation. Guatemala's priorities with regard to NTDs include controlling STH, trachoma, leishmaniasis, Chagas and continuing the efforts to eliminate onchocerciasis. Taking advantage of potential cross-cutting activities with the health sector and between other sectors this project will focus on synergies between health, housing improvements and 


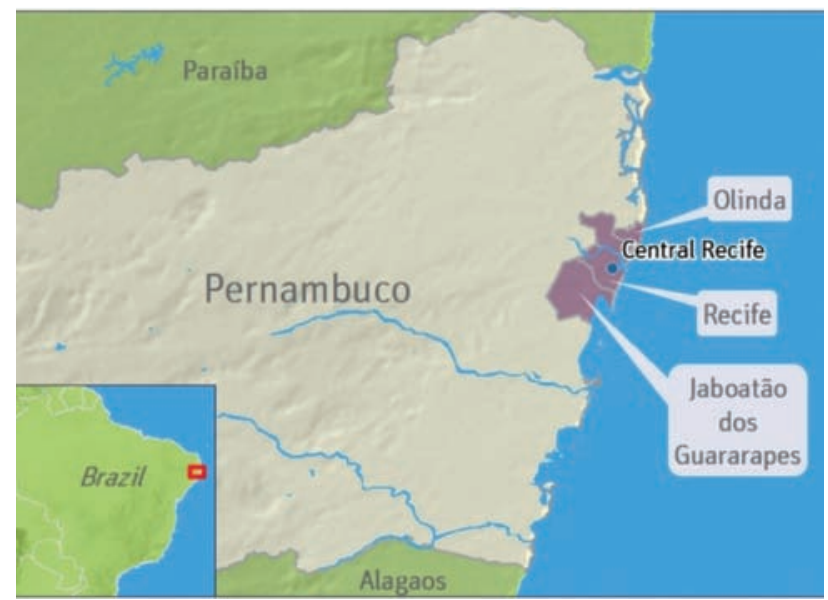

Fig. 2. IDB project RG-X1051: integrated control for NTDs in the municipalities of Recife, Olinda and Jaboatão dos Guararapes in the state of Pernambuco, Brazil.

water and sanitation. In addition, the strategy will include community health education through "The Healthy Family Model" - an innovative new approach to offering peer education to target audiences that has been developed especially for this project. This project seeks to benefit the 1.23 million people living in the targeted municipalities in the four prioritized departments in Guatemala that are at risk for STHs and are possibly at risk for trachoma, and other NTDs. Lives and livelihoods will be expected to improve, as a result of this work.

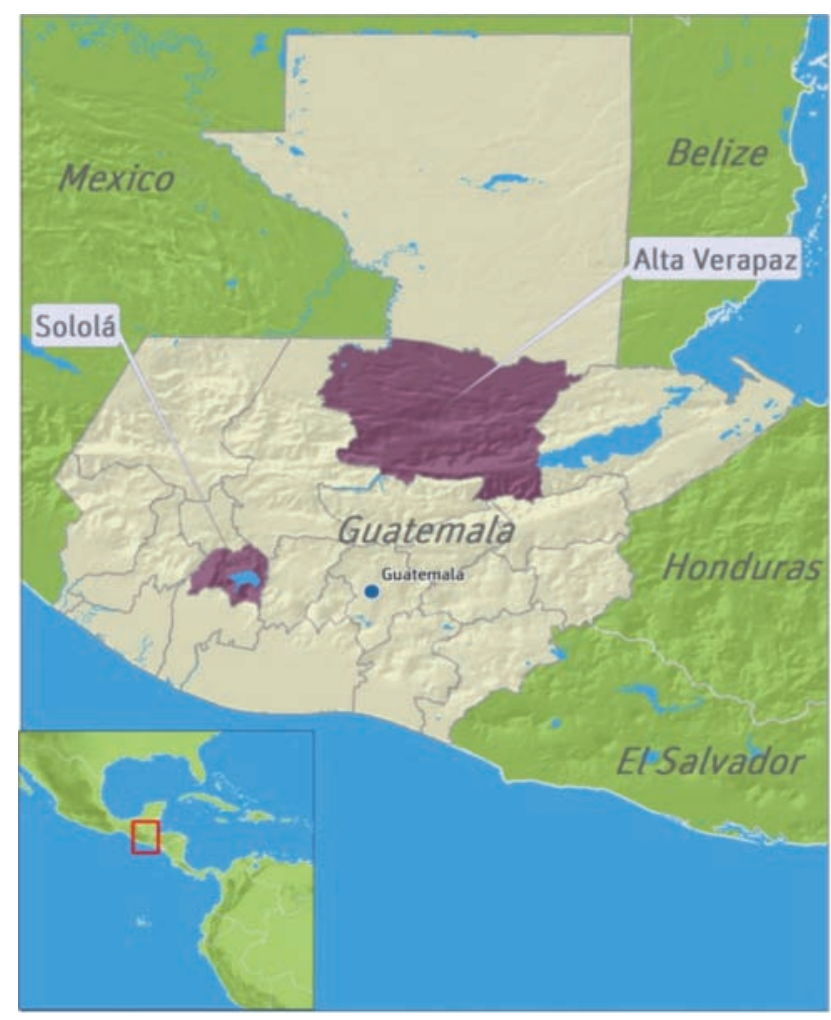

Fig. 3. IDB project GU-T1169: control of NTDs in Guatemala.

\section{Guyana, Demerara-Mahaica (region 4)}

LF and STH are both public health problems in Guyana and are amenable to control both by MDA and improvements in water and sanitation conditions. Guyana's capital city of Georgetown has an inadequate surface-water drainage system and is prone to severe and prolonged flooding during the rainy season. The aging sewerage system in Central Georgetown is prone to leaks and the electricity supply at its pumping stations is unstable. These problems cause wastewater to overflow into the drainage trenches and back-up into the streets and backyards. This in turn causes populations of Culex mosquitoes - a LF vector - and STH transmission to increase. The water and sanitation division of the IDB (INE/WSA) has proposed a programme to improve the sanitary conditions in Georgetown through the rehabilitation of the city's sewerage system. The IDB has therefore taken advantage of the opportunity to add a health component to this project in order to combine the impact of water and sanitation improvements with medical and public health interventions and eliminate LF and prevent transmission of STH in this region of 310,000 inhabitants (Fig. 4).

\section{Haiti, various locations}

LF and STH are both present in Haiti. Prior to the earthquake in January 2010, water and sanitation conditions in this impoverished country were already in an alarming state, with just $50 \%$ of urban residents and $30 \%$ of rural residents having access to potable water. Because of the devastating impact of the earthquake and its impact on the water and sanitation infrastructure, the inhabitants of Port-au-Prince and its surrounding areas have been forced to live in even more deplorable conditions. It is likely that the achievements made in controlling NTDs in Haiti in recent years will have been set back considerably by the disaster. In response, three projects will be implemented by the IDB to improve the water and sanitation services in Haiti (one in the capital, one in the neighbouring department of Artibonite and a third in five intermediate-sized cities) (Fig. 5) each of which will include an NTD component. In addition, deworming has been added to a package of services included in the national child health weeks, which are partly funded by IDB SPH.

\section{SPH's work in the geospatial health area}

The IDB's SPH division is increasingly relying on geospatial analysis techniques to support the analyti- 


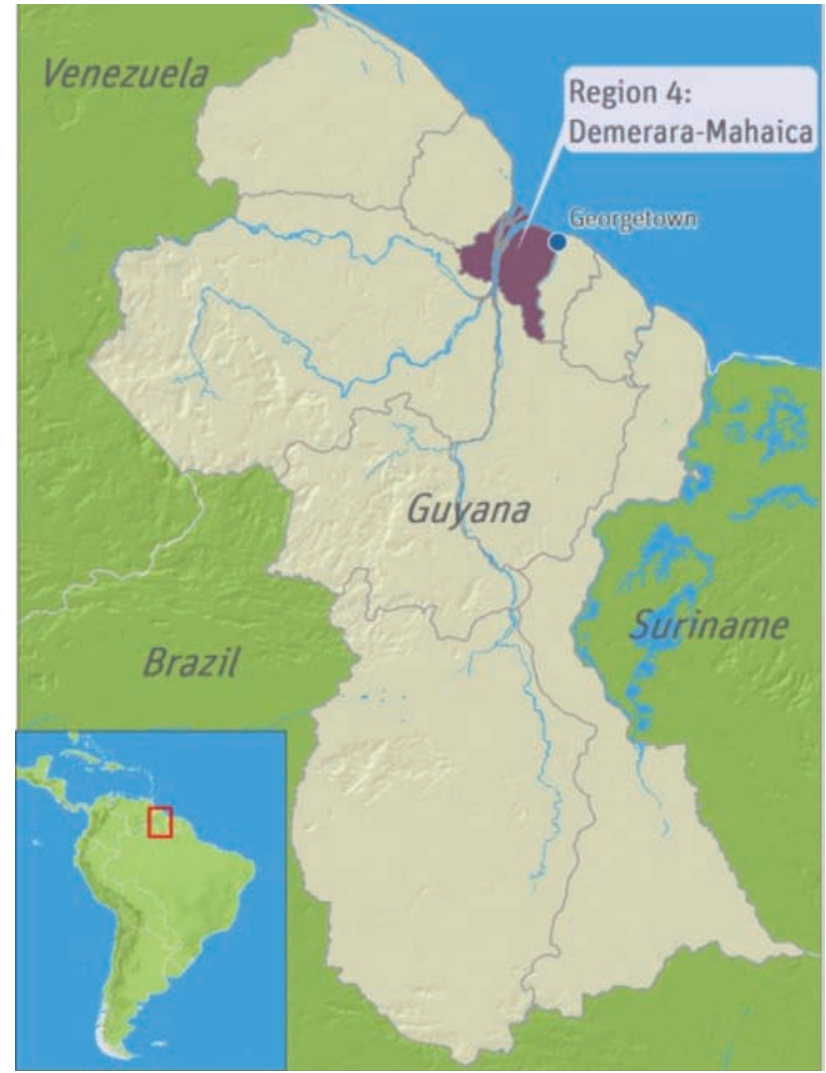

Fig. 4. IDB Project GY-L1025: the Georgetown sanitation improvement programme.

cal work on the NTDs and the Salud Mesoamerica 2015 initiatives as well as other projects. Geographical information systems (GIS) software and data have proven to be valuable tools for visualizing both geographical areas of intervention for the activities of the initiatives and the distribution of key variables of interest (such as poverty and NTD risk factors). Some of the activities that have been carried out in the last year include:

(i) the production of maps to show the geographical location of proposed IDB projects;

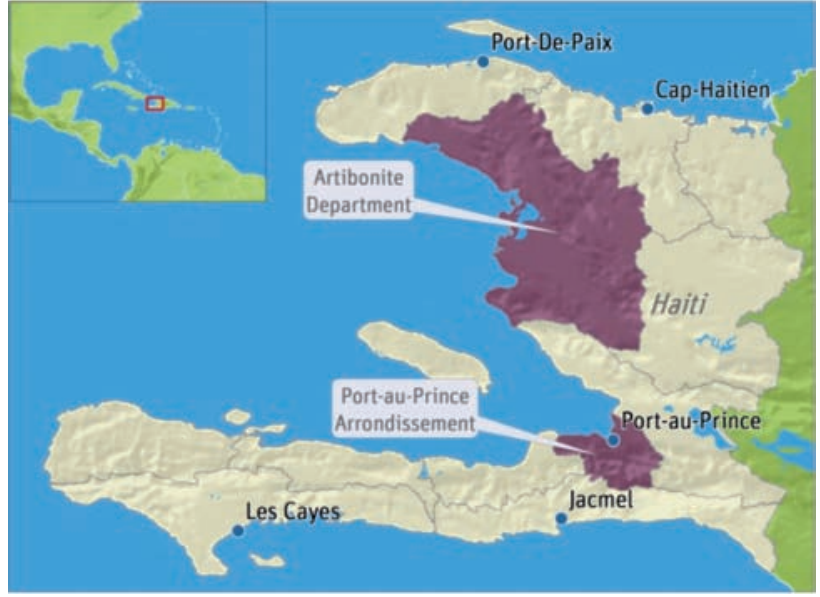

Fig. 5. IDB projects HA-X1024: integrated control for NTDs in Port-au-Prince; HA-L1044/HA-X1021 Port-au-Prince water and sanitation project; HA-X1014 rural water and sanitation program; HA-L1039/HA-X1013 water and sanitation for intermediate cities.

(ii) the mapping of poverty indicators at municipality level within countries to identify the areas that contain the poorest quintile of the population;

(iii) the mapping of five socio-economic risk factors for STH within the LAC region at the second level of administrative division. It is hoped that this data will later be used to construct an aggregate indicator of STH transmission in order to estimate the population at risk and the amount of resources required to control the disease;

(iv) the mapping of maternal health services in key priority municipalities in Honduras in order to show their proximity to the communities they serve (using buffers and Thiessen polygons); and

(v) the mapping of the distribution of NTDs for individual countries superimposed over the mapping of sanitation access at municipality level. 